\title{
Effect of Octreotide Injection on Postoperative Drainage After Neck Dissection: A Preliminary Report of a Prospective, Matched Case-Control Study
}

\author{
Dongbin Ahn ${ }^{1} \cdot$ Jae Han Jeon ${ }^{2} \cdot$ Heejin $\mathrm{Kim}^{1} \cdot$ Jin Ho Sohn ${ }^{1}$ \\ Departments of ${ }^{1}$ Otorhinolaryngology-Head and Neck Surgery; ${ }^{2}$ Internal Medicine, \\ Kyungpook National University School of Medicine, Daegu, Korea
}

Objectives. Somatostatin inhibits lymph production and reduces lymph flow into the lymphatic duct. We hypothesized that octreotide, a long-acting somatostatin analog, would reduce drainage after neck dissection (ND) by reducing the overall lymphatic flow in the neck as well as thoracic duct flow.

Methods. From 2012 to 2014, total 123 patients who had undergone left-sided comprehensive ND, were divided into an octreotide group (49 patients) and a control group (74 patients). Seventeen patients from the octreotide group and 17 from the control group were individually matched by age ( \pm 10 years), sex, body mass index $\left( \pm 1 \mathrm{~kg} / \mathrm{m}^{2}\right)$, type of cancer, surgeon, and the extent of surgery. These 34 patients were finally included in the study.

Results. The total fluid drainage volume $(540.9 \mathrm{~mL}$ vs. $707.9 \mathrm{~mL})$ and drainage volume during the period of octreotide use (the first 5 postoperative days) $(461.1 \mathrm{~mL}$ vs. $676.4 \mathrm{~mL})$ were significantly lower in the octreotide group. The duration of drain placement (6.3 days vs. 9.4 days) was also shorter in the octreotide group. In the octreotide group, the mean triglyceride concentration in the drainage fluid was significantly lower than that in the control group (43.1 mg/ $\mathrm{dL}$ vs. $88.8 \mathrm{mg} / \mathrm{dL}$ ). There was no complication associated with the use of octreotide.

Conclusion. Our study has shown that postoperative octreotide injections reduce postoperative drainage and the duration of drain placement. Further studies with larger patient populations are warranted to confirm these results and to evaluate the clinical benefits for patients.

Keywords. Chyle; Drainage; Neck Dissection; Somatostatin; Triglyceride

\section{INTRODUCTION}

Neck dissection (ND) as a therapeutic or elective intention is one of the most commonly used surgical procedures in the treatment of various head and neck cancers. The surgical technique has been well-standardized and has contributed to improved

\footnotetext{
- Received January 13, 2015

Revised June 22, 2015

Accepted July 22, 2015

- Corresponding author: Jin Ho Sohn

Department of Otolaryngology-Head and Neck Surgery, Kyungpook

National University Hospital, Kyungpook National University School of

Medicine, 130 Dongdeok-ro, Jung-gu, Daegu 41944, Korea

Tel: +82-53-200-2792, Fax: +82-53-423-4524

E-mail: entgodlikeu@gmail.com
}

disease control for patients with head and neck cancers [1,2]. However, ND, particularly comprehensive dissection, may be accompanied by postoperative morbidities associated with radical resection of tissue such as hematoma, seroma, chyle fistula, wound infection, and skin flap complications [3].To reduce these complications, negative pressure drainage systems are used for complete drainage of fluid collection [4]. Topical hemostatic agents including fibrin sealant or collagen sealant patches have also been widely used in various fields to reduce overall drainage [5-8]. However, benefits of these local materials is questionable because their mechanism of action depends mainly on mechanical sealing of transected tissue via an induced coagulation process, and they usually cannot cover the whole surgical bed in surgeries of comprehensive extent [5]. In fact, there are conflict-

Copyright $@ 2016$ by Korean Society of Otorhinolaryngology-Head and Neck Surgery

This is an open-access article distributed under the terms of the Creative Commons Attribution Non-Commercial License (http://creativecommons.org/licenses/by-nc/4.0)

which permits unrestricted non-commercial use, distribution, and reproduction in any medium, provided the original work is properly cited. 
ing trial results in terms of the efficacy of sealing agents for reducing postoperative drainage [9-11].

In contrast to the topical hemostatic materials, systemic administration of somatostatin can directly inhibit lymph production and reduce lymph flow into the lymphatic duct by inhibition of endocrine and exocrine secretions of the pancreas, intestine, and gallbladder, as well as by inhibiting nutrient absorption from the intestine and decreasing splanchnic blood flow [12-14]. On the basis of this premise, octreotide, a long-acting somatostatin analog, has been used as a medical treatment option for iatrogenic or congenital lymphorrhea, and has been described in most reports as highly effective with very few adverse effects [14-16]. Considering the theoretical mechanisms and clinical evidences, we hypothesized that octreotide would reduce drainage after ND by reducing overall lymphatic flow in the neck as well as thoracic duct flow, even in cases without chyle fistula.To date, there have been no studies concerning the effect of the routine use of octreotide to reduce drainage after ND. The primary objective of the present study was to determine whether octreotide would provide clinical reductions in postoperative drainage after comprehensive ND. The secondary objective was to evaluate the effect of octreotide on triglyceride (TG) concentrations of drainage, which would suggest a possible effect of octreotide on the nature of the drainage.

\section{MATERIALS AND METHODS}

\section{Study design and patients}

This study was designed as a prospective matched case-control study. The Institutional Review Board of Kyungpook National University Hospital approved the study protocol (registration number: 2012-09008), and written informed consent for the study was obtained from all patients.

We confined the extent of ND for eligibility to left-sided comprehensive ND including modified radical ND of level I to V or selective ND of level II to V considering the anatomical location of the major lymphatic duct in the lower neck, which terminates at the angle of the junction of the left internal jugular and subclavian veins [17].

From May 2012 to January 2014, total 123 patients who underwent comprehensive ND of the lateral compartment of the left neck for the management of head and neck cancer were enrolled in the study. After being provided with sufficient information about the possible benefits and risks of the octreotide administration, each patient selected his or her own study group before the surgery and was enrolled into either an octreotide group (49 patients) or a control group (74 patients). From these, 17 patients from the octreotide group and 17 from the control group who were individually matched by age ( \pm 10 years), sex, body mass index (BMI, $\pm 1 \mathrm{~kg} / \mathrm{m}^{2}$ ), type of cancer, surgeon, and extent of surgery were finally included in the study.
Exclusion criteria included previous ND, previous radiotherapy or chemotherapy, steroid medication within the past 6 months, anticoagulation medication, uncontrolled hyperlipidemia with preoperative fasting serum TG concentrations $>150$ $\mathrm{mg} / \mathrm{dL}$, and uncontrolled diabetes mellitus.

\section{Surgical technique}

All NDs were performed by 2 head and neck surgeons (DA and JHS). The harmonic scalpel is routinely used for tissue dissection and hemostasis; however, we utilized a conventional clamp-andtie technique for the dissection of the lower level IV region in concern of the thoracic duct fistula. All ND procedures were performed carefully, particularly around high risk areas for bleeding or chyle fistula. Dissection to identify the major lymphatic duct was not routinely used because the risk of iatrogenic injury to the duct might increase. At the completion of the dissection, all patients were carefully observed for potential chyle leakage. Each patient was placed in the Trendelenburg position, and the wound was observed during prolonged positive pressure ventilation. Routine blind suturing of soft tissue is not performed as long as intraoperative thoracic duct injury is unsuspected. After secure hemostasis, two closed-suction drains were placed and the wounds were closed layer by layer.

\section{Administration of octreotide and management of drains}

In the octreotide group, octreotide $0.1 \mathrm{mg}$ was administered subcutaneously immediately after surgery and repeated every 8 hours (3 times a day) until postoperative day (POD) 5 . All patients in the both groups resumed regular oral diets on the first day after surgery. The amount of fluid from the closed-suction drains was recorded daily at $6 \mathrm{AM}$ and the TG concentration in the drainage fluid was checked daily until POD 5. Biochemical chyle fistula was defined as TG concentration of drainage $>100$ $\mathrm{mg} / \mathrm{dL}$ without any clinical signs or symptoms of chyle fistula such as massive increase of drainage amount, milky drain production, and wound swelling while clinical chyle fistula was defined in the presence of clinical signs or symptoms of chyle fistula combined with TG concentrations of drainage fluid of $>100$ $\mathrm{mg} / \mathrm{dL}$. In cases of biochemical chyle fistula, only nutritional modification consisting of a medium chain TG diet was used and the TG concentration of the drainage fluid was monitored until it was stable within normal range. In cases of clinical chyle fistula, nutritional modification and pressure dressings were used.

When the total drain output decreased to $<15 \mathrm{~mL}$, the drain was removed. All patients were assessed twice daily, including inspection of wound and color of drainage. Laboratory studies including complete blood count, serum biochemistry, and lipid profile were obtained on POD 1, 3, and 5.

Patients were monitored for possible adverse events of octreotide use on the basis of history and physical examination as well as laboratory studies. Adverse events were graded using the Common Terminology Criteria for Adverse Events (CTCAE) 
ver. 4.0 (National Cancer Institute, Bethesda, MD, USA). If any grade 3-4 adverse events occurred during the period of octreotide administraion, the patients would be withdrawn from the study and appropriate management for the adverse events would be provided.

\section{Statistical analyses}

SPSS ver. 12.0 (SPSS Inc., Chicago, IL, USA) was used to analyze the data. Continuous data were presented as mean \pm standard deviation and were compared between groups using independent Student $t$-test. Categorical data were compared using chi-square test or Fisher exact test. Statistical significance was defined as $P<0.05$ and all $P$-values were two-sided throughout.

\section{RESULTS}

\section{Patient characteristics}

Since this study was designed as a matched case-control study to eliminate major confounding factors, there were no significant differences with respect to age, sex, body weight, BMI, type of cancer, and types of ND (Table 1). The mean number of harvested lymph node was also comparable between the 2 groups (49.4 \pm 28.1 vs. $48.7 \pm 21.6, P=0.706$ ). Although the mean number of positive lymph node was lower in the octreotide group compared to the control group, this difference was not statistically significant ( $6.3 \pm 6.4$ vs. $9.4 \pm 8.6, P=0.238$ ).

\section{Characteristics of postoperative drainage}

Postoperative drainage fluid volume, TG concentrations in the drainage fluid, and incidence of chyle fistula are listed in Table 2. Total drainage volume and drainage volume during POD 1-5 were significantly lower in the octreotide group compared to the control group $(540.9 \pm 308.5 \mathrm{~mL}$ vs. $807.9 \pm 342.4 \mathrm{~mL}, P=$ $0.025 ; 461.1 \pm 245.7 \mathrm{~mL}$ vs. $676.4 \pm 262.6 \mathrm{~mL}, P=0.02$, respectively). The duration of drain placement was also significantly shorter in the octreotide group compared to the control group (6.3 \pm 1.5 days vs. $9.4 \pm 4.1$ days, $P=0.013$ ).

Biochemical analysis of the drainage fluid showed that the mean TG concentrations during POD 1-5 was lower in the octreotide group than in the control group and this difference was statistically significant $(43.1 \pm 18.3 \mathrm{mg} / \mathrm{dL}$ vs. $88.8 \pm 64.2 \mathrm{mg} / \mathrm{dL}$,

Table 1. Characteristics of 34 patients enrolled into the study

\begin{tabular}{|c|c|c|c|}
\hline Characteristic & Octreotide group $(n=17)$ & Control group $(n=17)$ & $P$-value \\
\hline Age (yr) & $63.1 \pm 12.3$ & $59.0 \pm 18.0$ & 0.442 \\
\hline Sex (male:female) & $10: 7$ & $10: 7$ & - \\
\hline Body weight (kg) & $57.4 \pm 9.3$ & $60.5 \pm 10.2$ & 0.359 \\
\hline Body mass index $\left(\mathrm{kg} / \mathrm{m}^{2}\right)$ & $22.7 \pm 3.0$ & $22.8 \pm 2.8$ & 0.970 \\
\hline \multicolumn{4}{|l|}{ Disease } \\
\hline Thyroid cancer & 11 & 11 & - \\
\hline Laryngohypopharyngeal cancer & 2 & 2 & - \\
\hline Oral cavity-oropharygneal cancer & 2 & 2 & - \\
\hline Metastatic carcinoma of unknown primary site & 2 & 2 & - \\
\hline \multicolumn{4}{|l|}{ Type of neck dissection } \\
\hline SND (level II-V) & 11 & 11 & - \\
\hline mRND (level I-V) & 6 & 6 & - \\
\hline No. of harvested lymph nodes & $49.4 \pm 28.1$ & $48.7 \pm 21.6$ & 0.706 \\
\hline No. of positive lymph nodes & $6.3 \pm 6.4$ & $9.4 \pm 8.6$ & 0.238 \\
\hline
\end{tabular}

Values are presented as mean $\pm \mathrm{SD}$ or number.

SND, selective neck dissection; mRND, modified radical neck dissection.

Table 2. Characteristics of postoperative fluid drainage in control and octreotide treated patients.

\begin{tabular}{lccc}
\hline Characteristic & Octreotide group $(\mathrm{n}=17)$ & Control group $(\mathrm{n}=17)$ & $P$-value \\
\hline Total drainage volume $(\mathrm{mL})$ & $540.9 \pm 308.5$ & $807.9 \pm 342.4$ & $0.025^{*}$ \\
Drainage volume during POD 1-5 (mL) & $461.1 \pm 245.7$ & $676.4 \pm 262.6$ & $0.021^{*}$ \\
Duration of drain placement (day) & $6.3 \pm 1.5$ & $9.4 \pm 4.2$ & $0.013^{*}$ \\
Triglyceride concentration during POD 1-5 (mg/dL) & $43.1 \pm 18.3$ & $88.8 \pm 64.2$ & $0.034^{*}$ \\
Incidence of overall chyle fistula & $1(5.9)$ & $4(23.5)$ & 0.335 \\
Biochemical & $1(5.9)$ & $3(17.6)$ & 0.601 \\
Clinical & 0 & $1(5.9)$ & $>0.999$ \\
\hline
\end{tabular}

Values are presented as mean \pm SD or number (\%).

POD, postoperative day.

${ }^{*} P<0.05$, statistically significant differences between the groups. 
Table 3. Adverse events possibly related to octreotide use

\begin{tabular}{lccc}
\hline Adverse event & $\begin{array}{c}\text { Octreotide group } \\
(n=17)\end{array}$ & $\begin{array}{c}\text { Control group } \\
(n=17)\end{array}$ & $P$-value \\
\hline Nausea & $3(17.6)$ & $2(11.8)$ & $>0.999$ \\
Vomiting & $2(11.8)$ & $1(5.9)$ & $>0.999$ \\
Abdominal pain & $1(5.9)$ & $1(5.9)$ & $>0.999$ \\
Diarrhea & 0 & $1(5.9)$ & $>0.999$ \\
Hypoglycemia & $4(23.5)$ & $3(17.6)$ & $>0.999$ \\
Hyponatremia & $4(23.5)$ & $6(35.3)$ & 0.708 \\
Liver enzyme elevation & 0 & 0 & - \\
\hline
\end{tabular}

Values are presented as number (\%).

$P=0.034)$. In the octreotide group, only biochemical chyle fistula was found in 1 patient (5.9\%). In the control group, biochemical and clinical chyle fistulae were found in 3 patients $(17.6 \%)$ and 1 patient (5.9\%), respectively. These differences were not statistically significant $(P=0.335)$.

\section{Adverse events possibly related to octreotide use}

Table 3 lists the incidence of adverse events that were possibly related to octreotide use. There were no significant differences between the two groups in any types of adverse events. Hyponatremia (serum sodium concentration $<130 \mathrm{mmol} / \mathrm{L}$ ) and hypoglycemia (fasting serum glucose concentration $<55 \mathrm{mg} / \mathrm{dL}$ ) were the most common adverse events, occurring in 4 patients $(23.5 \%)$ of the octreotide group. However, these adverse events were also found in $6(35.3 \%)$ and 3 patients $(17.6 \%)$ of the control group, respectively. All adverse events were classified as grade 1 according to the CTCAE ver. 4.0 and all patients of the octreotide group completed the full course of intended postoperative administration of octreotide.

\section{DISCUSSION}

The primary aim of the present study was to investigate whether postoperative administration of octreotide would reduce postoperative drainage volume. We found that the postoperative fluid drainage volume reduced significantly in the octreotide group. Furthermore, the reduction in postoperative fluid drainage contributes to drain removal approximately 3 days earlier than that in the control group. Although 4 out of the 17 patients in the octreotide group showed a small reincrease in drainage volume after stopping octreotide injection, this increase was transient and the readministration of octreotide or any other forms of management was not required. We assumed that this result was associated with the effect of octreotide on the lymphatic system, i.e., octreotide reduces lymphatic production and flow. In the context of chyle fistula, the efficacy of somatostatin may be a result of its ability to reduce gastric, pancreatic, and intestinal secretion, to inhibit the motor activity of the intestine, ant to slow the process of intestinal absorption, reduce splanchnic blood flow, and decrease hepatic venous pressure $[13,16]$. In fact, a prospective study in dogs has demonstrated a threefold decrease in thoracic duct flow and decreased ratio of TG after administration of octreotide [18]. Similarly, in the present study, the TG concentrations in the postoperative drainage fluid were consistently lower in the octreotide group than in the control group throughout the postoperative period. These results suggest that both the lymph flow and TG concentrations in the major lymphatic channels were attenuated by octreotide injection, even though our study population did not initially included patients with chyle fistula. Furthermore, there was a trend toward a lower incidence of chyle fistula (biochemical or clinical) in the octreotide group. This finding also supports the hypothesized effect of octreotide on the lymphatic system and corresponds with the results of previous studies $[12,15,16]$.

Interestingly, the overall incidence of chyle fistula in the present study was $14.7 \%$ (5 of 34), which was considerably higher than that expected or described in the literature so far (reported range, $1 \%$ to $5.8 \%)[13,14,19]$. This may have been due to close prospective monitoring of our patients for potential chyle leakage. Although there have been no obvious signs or symptoms of chyle fistula to facilitate diagnosis during the postoperative period, routine biochemical assay of the drainage fluid may have affected the rate of clinical diagnosis. In a study by Roh et al. [17] regarding the prospective identification of chyle fistula in patients undergoing lateral ND for metastatic thyroid cancer, postoperative chyle fistula was observed in $8.3 \%$ patients, which was also higher incidence than that reported previously. Therefore, chyle fistula may be more frequent after ND than previously experienced, although only a minority of these patients are identified clinically and require active management. In fact, the incidence of clinical chyle fistula was only $2.9 \%$ (1 of 34 ) in our study, which is comparable to the previous studies.

As a preliminary study, the present study has some limitations that could be addressed by further investigation. First, the costeffectiveness and impact on quality of life due to repeated octreotide administration were not evaluated in the present study. We assume that earlier patient discharge would result from reduced fluid drainage volume and could contribute to reducing overall medical costs and an earlier return to routine social life. However, these benefits should be weighed against the additional costs and discomfort associated with multiple octreotide injections. Second, considering that the postoperative fluid collection is a consequence of surgical disruption of lymphatics and capillaries with ensuing leakage of fluid into the dead space created by surgical dissection [20], combining use of octreotide to reduce systemic lymphatic flow and a topical hemostatic agent to reduce the volume of dead space and seal leaking capillaries and lymphatic channels would theoretically be more powerful methods. To verify this, prospective randomized controlled trials including control group, a topical hemostatic agent group, a systemic octreotide group, and a topical hemostatic agent + sys- 
temic octreotide group should be performed.

In conclusion, this prospective matched case-control study has shown that postoperative octreotide injection in patients undergoing left-sided comprehensive ND reduces postoperative drainage volume and TG concentration in drainage fluid, resulting in earlier removal the drain without any adverse events. Further studies with larger patient populations are warranted to confirm these results and to evaluate the clinical benefits for patients.

\section{CONFLICT OF INTEREST}

No potential conflict of interest relevant to this article was reported.

\section{ACKNOWLEDGMENTS}

This work was supported by Biochemical Research Institute grant, Kyungpook National University Hospital (2013), Republic of Korea.

\section{REFERENCES}

1. Givi B, Linkov G, Ganly I, Patel SG, Wong RJ, Singh B, et al. Selective neck dissection in node-positive squamous cell carcinoma of the head and neck. Otolaryngol Head Neck Surg. 2012 Oct;147(4):70715.

2. Strong EW. Preoperative radiation and radical neck dissection. Surg Clin North Am. 1969 Apr;49(2):271-6.

3. Byers RM, Ballantyne AJ, Goepfert H, Guillamondegui OM, Larson DL, Medina J. Clinical effects of closed suction drainage on wound healing in patients with head and neck cancer. Arch Otolaryngol. 1982 Nov;108(11):723-6.

4. Durai R, Mownah A, Ng PC. Use of drains in surgery: a review. J Perioper Pract. 2009 Jun;19(6):180-6.

5. SpotnitzWD. Fibrin sealant: past, present, and future: a brief review. World J Surg. 2010 Apr;34(4):632-4.

6. Ochiai T, Sonoyama T, Soga K, Inoue K, Ikoma H, Shiozaki A, et al.
Application of polyethylene glycolic acid felt with fibrin sealant to prevent postoperative pancreatic fistula in pancreatic surgery. J Gastrointest Surg. 2010 May;14(5):884-90.

7. Di Monta G, Caraco C, Crispo A, Marone U, Mozzillo N. Collagen sealant patch to reduce lymphatic drainage after lymph node dissection. World J Surg Oncol. 2012 Dec;10:275.

8. Vida VL, Padalino MA, Barzon E, Stellin G. Efficacy of fibrinogen/ thrombin-coated equine collagen patch in controlling lymphatic leaks. J Card Surg. 2012 Jul;27(4):441-2.

9. Figueras J, Llado L, Miro M, Ramos E, Torras J, Fabregat J, et al. Application of fibrin glue sealant after hepatectomy does not seem justified: results of a randomized study in 300 patients. Ann Surg. 2007 Apr;245(4):536-42.

10. Lee MG, Jones D. Applications of fibrin sealant in surgery. Surg Innov. 2005 Sep;12(3):203-13.

11. Swan MC, Oliver DW, Cassell OC, Coleman DJ,Williams N, Morritt DG, et al. Randomized controlled trial of fibrin sealant to reduce postoperative drainage following elective lymph node dissection. $\mathrm{Br}$ J Surg. 2011 Jul;98(7):918-24.

12. Ulibarri JI, Sanz Y, Fuentes C, Mancha A, Aramendia M, Sanchez S. Reduction of lymphorrhagia from ruptured thoracic duct by somatostatin. Lancet. 1990 Jul;336(8709):258.

13. Nussenbaum B, Liu JH, Sinard RJ. Systematic management of chyle fistula: the Southwestern experience and review of the literature. Otolaryngol Head Neck Surg. 2000 Jan;122(1):31-8.

14. Valentine CN, Barresi R, Prinz RA. Somatostatin analog treatment of a cervical thoracic duct fistula. Head Neck. 2002 Aug;24(8):8103.

15. Szabados E, Toth K, Mezosi E. Use of octreotide in the treatment of chylopericardium. Heart Lung. 2011 Nov-Dec;40(6):574-5.

16. Coskun A, Yildirim M. Somatostatin in medical management of chyle fistula after neck dissection for papillary thyroid carcinoma. Am J Otolaryngol. 2010 Sep-Oct;31(5):395-6.

17. Roh JL, Kim DH, Park CI. Prospective identification of chyle leakage in patients undergoing lateral neck dissection for metastatic thyroid cancer. Ann Surg Oncol. 2008 Feb;15(2):424-9.

18. Nakabayashi H, Sagara H, Usukura N, Yoshimitsu K, Imamura T, Seta T, et al. Effect of somatostatin on the flow rate and triglyceride levels of thoracic duct lymph in normal and vagotomized dogs. Diabetes. 1981 May;30(5):440-5.

19. Gregor RT. Management of chyle fistulization in association with neck dissection. Otolaryngol Head Neck Surg. 2000 Mar;122(3): 434-9.

20. Woodworth PA, McBoyle MF, Helmer SD, Beamer RL. Seroma formation after breast cancer surgery: incidence and predicting factors. Am Surg. 2000 May;66(5):444-50. 